\title{
Impact of Different Spacing Policies for Adaptive Cruise Control on Traffic and Energy Consumption of Electric Vehicles
}

\author{
Bilgehan Bayar ${ }^{1}$, S. Amin Sajadi-Alamdari ${ }^{2}$, Francesco Viti ${ }^{3}$ and Holger Voos ${ }^{3}$
}

\begin{abstract}
This paper assesses the impact of different spacing policies for Adaptive Cruise Control (ACC) systems on traffic and environment. The largest deal of existing studies focus on assessing the performance in terms of safety, while only few deal with the effect of ACC on the traffic flow and the environment. In particular, very little is know on traffic stability and energy consumption. In this study, the vehicles equipped with ACC are modelled and controlled by two different spacing policies. Besides, Human Driving Behavior (HDB) is modelled by using Gipps model for comparison and for simulating different penetration rates. As distinguished from other studies, vehicle dynamics and energy consumption of an electric car is formulated, which has completely different characteristics and limitations than combustion engine cars. Hence the study aims at providing additional understanding of how ACC-equipped electric vehicles will behave in dense traffic conditions. HDB and ACC vehicles are placed in a roundabout at different penetration rates. String stability and energy consumption are investigated by giving a shock wave to a stable traffic condition. It is found that ACC with quadratic spacing policy has significantly positive effects on string stability and energy consumption.
\end{abstract}

\section{INTRODUCTION}

As vehicle technology advances every day, the safety and comfort of passengers improve and the number of produced vehicles arises. However, traffic problems also emerge as one of the biggest issues of the century with increasing vehicle population. Even though vehicles are getting smarter with advancing technology, one simply cannot foresee the positive effects on traffic; to improve the current traffic situation, systematic and futuristic traffic control strategies are needed for intelligent vehicles. Therefore, researchers [1], [2] are seeking solutions to achieve intelligent transportation systems, and they believe that improvements in the vehicle automation and communication systems can play an important role in addressing the issues of traffic.

These considerations have initiated much research into vehicle automation. The adaptive cruise control (ACC) system is one of the most commercialized ones and can change the dynamic of traffic significantly. ACC is an advanced cruise control system and compared to a conventional cruise system, which sets only cruising speed, it tries to maintain a desired speed and a desired following distance with a

\footnotetext{
${ }^{1}$ Bilgehan Bayar, M.Sc. student at Faculty of Science, Technology and Communication, University of Luxembourg, Luxembourg-City, L-1359 Luxembourg bilgehan.bayar.001@student.uni.lu

${ }^{2} \mathrm{~S}$. Amin Sajadi-Alamdari, Ph.D. candidate at Interdisciplinary Centre for Security, Reliability and Trust, University of Luxembourg, LuxembourgCity, L-1359 Luxembourg

${ }^{3}$ Francesco Viti and Holger Voos, professor at Faculty of Science, Technology and Communication, University of Luxembourg, LuxembourgCity, L-1359 Luxembourg
}

vehicle in front [3]. Such systems have been widely studied and different mathematical models have been developed according to their research purpose. However, only little research exist on the behaviour of electric vehicles, even though are seen as future of the transportation.

The first ACC systems were introduced to decrease the responsibility and stress on drivers so that they can focus on more important tasks like steering during the journey. In this way, the comfort and safety of the driving have been increased [4]. Moreover, researchers attach much importance to improving traffic by using ACC systems and proved that string stability and tighter spacing policy can be achieved by increasing the percentage of vehicles assisted by ACC in the traffic [1], [2], [5], [6], [7]. String stability and spacing policy are important to solve traffic problems. String stability is directly affected by applied spacing policies between vehicles, whereas unstable string behavior may cause traffic jams. Unstable string behavior can be observed without any cause such as an accident or a construction, basically it is caused by human driving behaviors in the traffic system [8].

In this paper, a control law for an ACC system [9] with two different spacing policies is considered to evaluate the effects on traffic characteristics. The first spacing policy is linear, which is already developed and used for the chosen control law in some literatures [9], [10], and its string stability is proven after "the infimal time headway" value [11]. The second one is nonlinear with a quadratic term for vehicle speed whose parameters are optimized for traffic flow stability and string stability [12]. Besides, human driving behavior is considered in comparison with the ACC driving models. Human driving behavior is predicted by using the Gipps model [13], and the parameters in the model are chosen according to the statistical study on a modified version of the Gipps model [14], This paper mainly focuses on string stability and traffic flow capacity, yet the traffic flow stability, which is another phenomenon about traffic stability [15], is not within the scope of this study.

The main objective of this work is to extend and evaluate the currently available understanding of the automotive control system's spacing policy. The selection of spacing policy has significant impacts on traffic performance regarding to traffic output, fuel/energy consumption, safety and so on. The need of such a study is notably stated before as a topic for future research [16]. For this purpose, quadratic spacing policy [12] is implemented in the chosen ACC model [9] and a proposed mathematical model for the application is explained in the next 'Control Algorithms' section. The application is modeled and simulated in a computer environment using 
Simulink (MathWorks) to evaluate the effects of the proposed model. The models (the current ACC, the proposed ACC and human driving behavior) are combined at different penetration rates in an assumed traffic situation, and their effects on string stability and traffic density are compared. In the 'Results \& Discussion' section, the simulation results are presented to state the differences in the models. Moreover, this paper provides an understanding of electric vehicle's dynamics and energy consumption, and their cooperation with ACC systems is analysed. An ACC-equipped electric vehicle's dynamic behaviors and energy consumption are formulated and applied in the traffic simulation. In that, only little research exist on the behaviour of electric vehicles, even though they are seen as future of transportation.

In addition to trying to solve the congestion issues and increase the density of traffic, this paper considers the total energy consumption of traffic according to the aforementioned driving models on a microscopic scale. For the consumption calculation, each vehicle in the traffic simulation is considered simultaneously under assumed situations. To calculate the total energy consumption per vehicle, a consumption formula and vehicle dynamics are calibrated on a Smart Electric Drive(ED), Model 2012, which is already derived by the researchers at the University of Luxembourg [17], [18].

\section{Control Algorithms}

Various control laws exist for ACC-equipped vehicles, though they have the same objective, to maintain safety distance while aiming at keep speeds near the desired ones, there are some small but very important differences. These differences directly affect vehicle and traffic dynamics. In this study, an ACC control law, which was tested before on Nissan cars, is chosen to work on [9]. Compared to the others, this model is chosen because of lower fluctuation and calculation's simplicity. The model has two different modes (speed control and gap control), the appropriate one is chosen regarding the instant distance with the followed vehicle. Speed control is used when the gap with the followed vehicle is greater than 120 meters, and it switches to gap control when the gap is less than 100 meters. For the values between 100 meters and 120 meters, the former control strategy is maintained to avoid any uncertainty during the transition process.

Speed control:

$$
\begin{gathered}
v_{e}=v-v_{d} \\
a_{s c}=\operatorname{bound}\left(-0.4 v_{e}, a_{\text {max }}, b_{\text {max }}\right) \\
a=a_{s c}
\end{gathered}
$$

Gap control:

$$
\begin{gathered}
v_{e}=v-v_{d} \\
a_{s c}=\operatorname{bound}\left(-0.4 v_{e}, a_{\max }, b_{\max }\right) \\
s_{d}=T_{d} \cdot v \\
s_{e}=s-s_{d}
\end{gathered}
$$

$$
a_{s c}=\operatorname{bound}\left(\dot{s}+0.25 s_{e}, a_{s c}, b_{\max }\right)
$$

where bound is defined as:

$$
\operatorname{bound}\left(x, x_{u b}, x_{l b}\right)=\max \left(\min \left(x, x_{u b}\right), x_{l b}\right)
$$

where $v$ is the speed of the vehicle equipped with $\mathrm{ACC}$, $v_{d}$ is desired speed given by the driver or the speed limit, $v_{e}$ is speed error, $a_{s c}$ is the acceleration in speed control mode, $s$ is the distance between the vehicle equipped with ACC and the preceding vehicle, $s_{e}$ is the spacing error, $s_{d}$ is the desired distance and $T_{d}$ is the time headway value. Finally, $a_{\max }$ and $b_{\max }$ are the maximum acceleration and maximum deceleration which can be provided by the car. These values are modified according to the chosen vehicle's dynamic characteristics which is explained in detail in the 'Consumption Modelling and Vehicle Dynamics' section.

The model has a linear spacing policy with a constant time headway parameter for the desired spacing gap calculation. For the linear spacing policy, string stability is proven after the critical time headway value of ' 1.47 ' [11]. In this study, a value above the critical value is chosen (10).

Besides the linear spacing policy, a quadratic spacing policy is implemented in the model for the desired distance calculation. The applied nonlinear range policy [12] has proven string and traffic stability above the critical velocity value $(13.4 \mathrm{~m} / \mathrm{s})$. Differences between policies can be seen in Fig. 1.

Linear spacing policy:

$$
s_{d}=1.5 v
$$

Quadratic spacing policy:

$$
s_{d}=3+0.0019 v+0.0448 v^{2}
$$

The deficiencies of the linear policy, poor robustness against traffic fluctuations, are tried to be resolved by replacing it with a quadratic spacing policy. Besides, the lower range values, when the quadratic spacing policy is used, are expected to increase the traffic capacity.

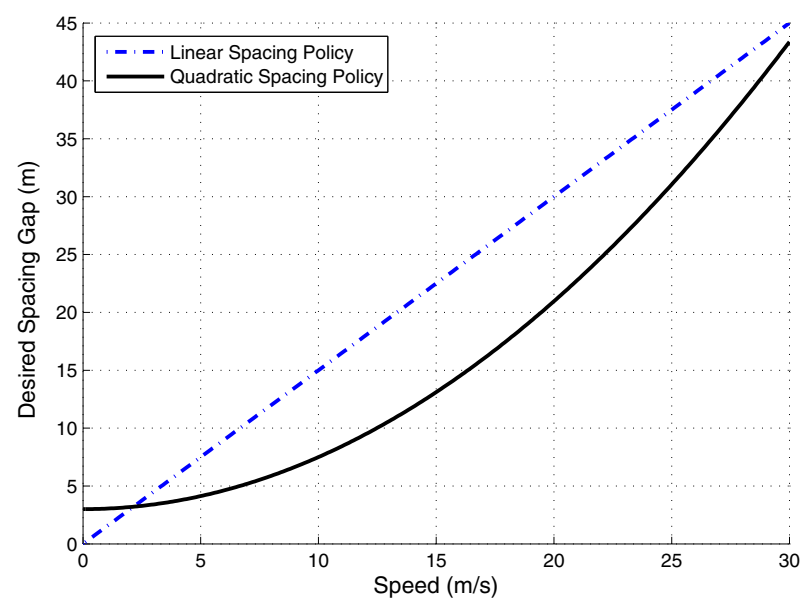

Fig. 1. Desired spacing gap-speed chart 


\section{Modelling Human Drivers}

Although driving behavior differs from one driver to another, plenty of researchers attempt to derive a common model for car-following [13], [19], [20]. Gipps is one of the pioneers and had been able to identify and model this behavior realistically [13], and other researchers modified the original model based on field optained car-following data [14]. In this study, the Gipps model is used to predict human behaviors in traffic. Parameters used for the model are chosen accordingly to the statistical distribution done for the modified Gipps model [14], and they can be found in Table I.

$$
\begin{array}{r}
v(t+\tau)=\min \left\{v_{n}(t)+2.5 a_{n} \tau\left(1-v_{n}(t) / V_{f}\right) *\right. \\
\left(0.025+v_{n}(t) / V_{f}\right)^{1 / 2}, b_{n} \tau+\left[\left(b_{n} \tau\right)^{2}+b_{n}\left[2 \left(x_{n-1}(t)\right.\right.\right. \\
\left.\left.\left.-R_{\min }-x_{n}(t)\right]-v_{n}(t) \tau-v_{n}(t)^{2} / \widehat{b}\right]^{1 / 2}\right\}
\end{array}
$$

TABLE I

Parameters Used for the GipPS MOdel

\begin{tabular}{cccc}
\hline \hline Symbol & Description & Value & Unit \\
\hline$a_{n}$ & Peak acceleration & 0.7664 & $\mathrm{~m} / \mathrm{s}^{2}$ \\
$V_{f}$ & Free-flow velocity & 30 & $\mathrm{~m} / \mathrm{s}$ \\
$b_{n}$ & Peak deceleration & -3.5388 & $\mathrm{~m} / \mathrm{s}^{2}$ \\
$\widehat{b}$ & Estimated peak deceleration & -3.0 & $\mathrm{~m} / \mathrm{s}^{2}$ \\
$R_{\text {min }}$ & Separating distance at standstill & 3.5094 & $\mathrm{~m}$ \\
$\tau$ & Apparent reaction time & 0.67 & $\mathrm{~s}$ \\
\hline \hline
\end{tabular}

$\widehat{b}$ value is chosen bigger than $b_{n}$ to propagate the disturbance and amplify the flow, as aim of human driving in this study is to model unstable human driving behavior and show how string stability can be achieved by penetration of ACC vehicles.

\section{Consumption Modelling And Vehicle DYNAMICS}

The ACC systems generally have been designed based on vehicle in a line or platoon following with respect to preceding vehicles. The vehicle longitudinal dynamics has been extensively studied for decades and greatly improve our understanding of vehicle control. Relatively less research is however found on the behaviour of electric vehicles equipped with ACC. The Smart ED has power train includes a lithiumion battery as the energy source, an electric machine, a single ratio transmission, and power electrics. In this paper, a simplified longitudinal vehicle dynamics for the Smart $E D$, and its energy consumption are modelled in order to evaluated the ACC system.

The longitudinal vehicle dynamics can be described as the Newton's second law in one-dimensional mass point motion as follows:

$$
\frac{d v}{d t}=\frac{F_{t r a c}-F_{d}-F_{r}}{M}
$$

where $F_{\text {trac }}$ is the traction force, $F_{d}$ is the aerodynamic drag resistance force, $F_{r}$ is the rolling resistance force, and $M$ is the vehicle's equivalent mass. It is noteworthy that road grade is not considered in this study. The traction force is controlled by the ACC system which is the control input for electric motor. The aerodynamic drag force is the resistive reaction of the vehicle's motion with air. This force is formulated as a speed based function follows by:

$$
F_{d}=\frac{1}{2} \rho A_{f} C_{d} v^{2}
$$

where $\rho$ is the air density, $A_{f}$ is the vehicle front area, $C_{d}$ is the aerodynamic drag coefficient. The rolling resistance results from the interactions between the tire and road surface. When the road angle is small, this force can be described in a simple form as

$$
F_{r}=\mu M g
$$

where $\mu$ is the rolling resistance coefficient, and $g$ is the gravitational constant. The equivalent mass of the vehicle increases due to the angular momentums of rotational parts (for more details see [17]). The traction power of the Smart ED is limited to its power train and brake systems. This limitation can be identified based on a Dynamo-meter test in order to measure the vehicle's traction force. The measurement data can help to describe the maximum and minimum limits of traction force mathematically as a speed related function as follows:

$$
F_{\text {trac }}=a \cdot \sin (b \cdot v+c)+d \cdot \sin (e \cdot v-f)
$$

where $a=4.0758, b=0.03043, c=2.182, d=0.2634, e=0.2368$, and $f=0.1372$ are the numerical constants.

The energy consumption of the vehicle is roughly affected by the driven speed and total forces acting on the vehicle. The deterministic approach to model the energy consumption of the electric vehicle can be a challenging task due to complexity of the energy path from the battery to tractive effort on the driven wheel associated with different road conditions. In this paper, however, the Dynamo-meter measurement from the energy consumption of the vehicle can be evaluated based on the traction force and speed characteristic map. Fig. 2. shows the power consumption of the electric vehicle (for more details see [17], [18]).

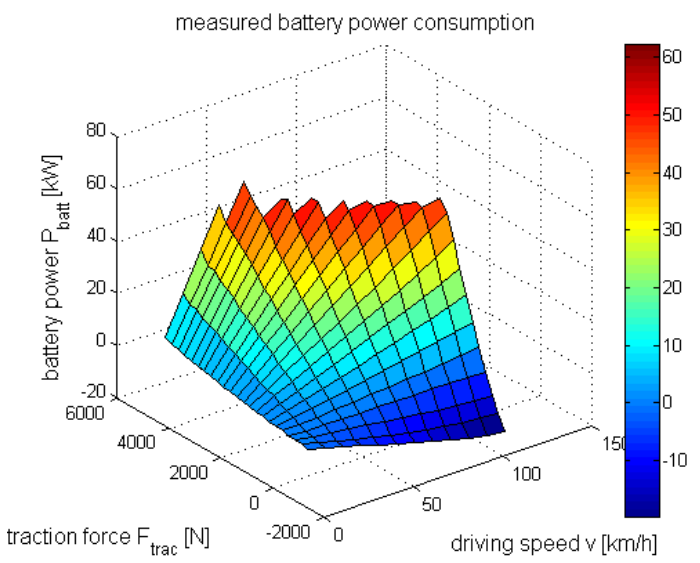

Fig. 2. Smart ED power consumption [17]. 
Hence, the power consumption, $P$, can be mathematically approximated as a bilinear relation of traction force and speed with $99.62 \%$ fitted regression line as follows:

$$
P=a \cdot v+b\left(F_{t r a c} v\right)+c \cdot F_{t r a c}
$$

where $a=223.3, b=1.059$, and $c=0.8141$ are the numerical constants. Note that the $F_{\text {trac }}$ is limited between $-1000(N)$ and $3000(N)$ as well as that the speed $v$ of the Smart ED is limited to $120(\mathrm{~km} / \mathrm{h})$.

\section{Simulation}

To simulate traffic and assess its stability, 20 vehicles are simulated in a circle where each vehicle follows the one in front, including the first and the last car. Hereby, the simulation track is formed into a circular road, experiments involving vehicles driving along a circle are rather traditional since the well known experiment from [8]. The curvature of the road is not taken into account as steering is considered not affecting the longitudinal driving behaviour. The closed circle simulation scenario with an adequate number of cars is chosen to exhibit the dynamics of real-life dense highway traffic network, which may become unstable by a small disturbance. Each vehicle is equipped with one of the control models, human driving behavior (HDB) or ACC algorithm which uses one of the following policies, either linear or quadratic. Human drivers and ACC-equipped vehicles, which use the same spacing policy, are combined at different penetration rates $(0 \%, 20 \%, 40 \%, 60 \%, 80 \%, 100 \%)$ to produce a realistic scenario which ACC and human drivers take place in the same traffic network. Each spacing policy is blended separately with HDB to exhibit the differences between them explicitly, especially to observe their compatibility at which penetration rate.

The simulation is started in a flowing traffic situation, each car has a speed of $90 \mathrm{~km} / \mathrm{h}$ and cars are placed in a roundabout with the same spacing gap. The first car is commanded with $90 \mathrm{~km} / \mathrm{h}$ for the first 70 seconds, and others are following it while they are allowed to increase their speed up to $110 \mathrm{~km} / \mathrm{h}$. It should be kept in mind that each control model has different following policy, especially human driving behavior has significantly shorter range behavior compared to ACC. This period is given to the vehicles to obtain the desired spacing gap and achieve a steady traffic flow situation. Thereby, the cars, which have a tighter spacing policy, can speed up for a while and decrease the initially given spacing gap. After obtaining the steady state flow, shockwave is applied to the leading car for 4 seconds at a rate of $-2 \mathrm{~m} / \mathrm{s}^{2}$, which decreases its speed to $68 \mathrm{~km} / \mathrm{h}$, then acceleration at a rate of $1 \mathrm{~m} / \mathrm{s}^{2}$ is given to reach the steady speed $(90 \mathrm{~km} / \mathrm{h})$ again. In real life this shock affect can be caused any kind of sudden break, such as unexpected object in front of a car.

\section{Results \& Discussion}

Figure 3 shows three mere control models representing the position of the vehicles in time, starting from $50^{\text {th }}$ second to $150^{\text {th }}$. Each line represents one car. The given shock wave at $70^{\text {th }}$ second affects drastically the vehicles controlled by Gipps model. Deceleration of the first car is followed by others increasingly and disturbance is propagated. As a result, the last car is stopped (Tab.II), it can also be seen in Fig. 3 the last car has zero slopes. Besides, a stop-and-go effect is also visible at Gipps model, a second shock wave is formed by itself around $90^{t h}$ second because of the fact that the decreased space between the car in front and at the end as it stopped for a while.

On the other hand, ACC-equipped vehicles perform a better reaction for the same shockwave and the disturbance is damped. A small change in the first cars' slope can be seen in Fig. 3 at $70^{\text {th }}$ second, which indicates the given shockwave, but the effect is absorbed by the following cars. Smoother traffic flow and less shock wave, which are the main constraints for string stability [21], are achieved for both of the models. As a result of the shockwave, the $20^{t h}$ car equipped with ACC with linear spacing policy decreased its speed to $82 \mathrm{~km} / \mathrm{h}$ and the $20^{\text {th }}$ car equipped with ACC with the quadratic spacing policy decreased its speed only to $85 \mathrm{~km} / \mathrm{h}$ (Table II and Fig. 4). The cars with quadratic spacing policy absorb the shockwave better than the cars with the linear spacing policy.

Traffic capacity is also increased with the quadratic spacing policy as expected. Higher intensity of the cars with the quadratic spacing policy can be seen in Fig. 3, it has darker color compared to the linear policy because of higher concentration of the cars. The cars with the linear spacing policy have $37.5 \mathrm{~m}$ range gap with the car in front at the speed of $90 \mathrm{~km} / \mathrm{h}$ whereas the cars with the quadratic spacing policy have $31 \mathrm{~m}$ range gap, which is almost $20 \%$ improvement. It increases traffic capacity at the same rate

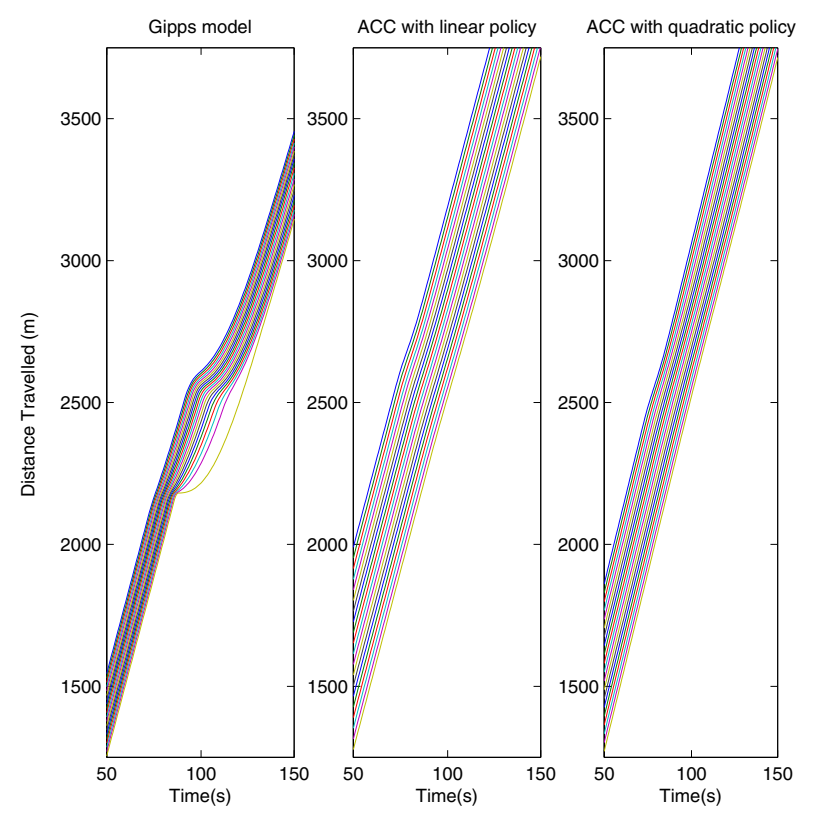

Fig. 3. Position-time chart of the cars at mere penetrations 
TABLE II

The Lowest Speed of the Last CAR After the Shock WaVe

\begin{tabular}{ccc}
\hline \hline \% of cars equipped with ACC & Linear & Quadratic \\
\hline \hline $100 \%$ & $82 \mathrm{~km} / \mathrm{h}$ & $85 \mathrm{~km} / \mathrm{h}$ \\
$80 \%$ & $78 \mathrm{~km} / \mathrm{h}$ & $82 \mathrm{~km} / \mathrm{h}$ \\
$60 \%$ & $72 \mathrm{~km} / \mathrm{h}$ & $78 \mathrm{~km} / \mathrm{h}$ \\
$40 \%$ & $60 \mathrm{~km} / \mathrm{h}$ & $68 \mathrm{~km} / \mathrm{h}$ \\
$20 \%$ & $28 \mathrm{~km} / \mathrm{h}$ & $28 \mathrm{~km} / \mathrm{h}$ \\
$0 \%$ & $0 \mathrm{~km} / \mathrm{h}$ \\
\hline \hline
\end{tabular}

while improving the string stability.

Three situations, which consist of mere control models, are displayed in Fig. 3 to observe distinctive differences between the models, yet traffic is composed of ACC and human drivers in real life. For this reason, ACC and HDB are combined at different penetration rates. The results of these simulations, the lowest speed of the last car in the circle after the shockwave, are shown in Table II. The results show that the last vehicles with the quadratic spacing policy slow down their speed less than the linear spacing policy, after the certain amount of ACC penetration in traffic (40\%). This result verifies that the quadratic policy ensures smoother traffic flow and less shock wave which leads to better string stability characteristics.

As $40 \%$ penetration is the critical situation, its speedtime chart for each car represented by a line is given in Fig. 4. The lowest speed of the first car (blue bold line) and the last car (red bold line) are equal to each other (68 $\mathrm{km} / \mathrm{h}$ ) at $40 \%$ penetration of the ACC with the quadratic spacing policy, which means $40 \%$ existence of the ACC can ensure string stability. Whereas the last car's speed has a significantly lower value $(60 \mathrm{~km} / \mathrm{h})$ with the linear spacing policy at the same penetration rate. Formation of a small second shockwave is visible as well in Fig. 4 for the linear spacing policy, the first car (blue bold line) decelerates around $95^{t h}$ second due to the fact that its reduced distance with the one at the tail. It may cause other shock waves or even a so called phantom traffic jam in highly dense traffic conditions. To provide the string stability higher amount of penetration is required for the linear spacing policy, such as $60 \%$ where the last car's speed $(72 \mathrm{~km} / \mathrm{h})$ is higher than the first one. On the other hand at low penetration rates $(20 \%)$ both policy have same lowest speed $(28 \mathrm{~km} / \mathrm{h})$ and string stability is not preserved as HDB is dominant.

Table III shows the percentage of energy saved by all cars in the simulations for the same travelled distance. Energy calculations are started from $70^{\text {th }}$ second to skip the initial speed-ups for reaching the desired range and finished as soon as the steady speed is obtained and same distance is travelled for all the models. Two mere ACC simulation's total energy consumptions are compared with the mere HDB simulation. Cars energy consumption is calculated individually and aggregated for the total consumption. The last ten cars total energy consumptions are also summed up and compared as
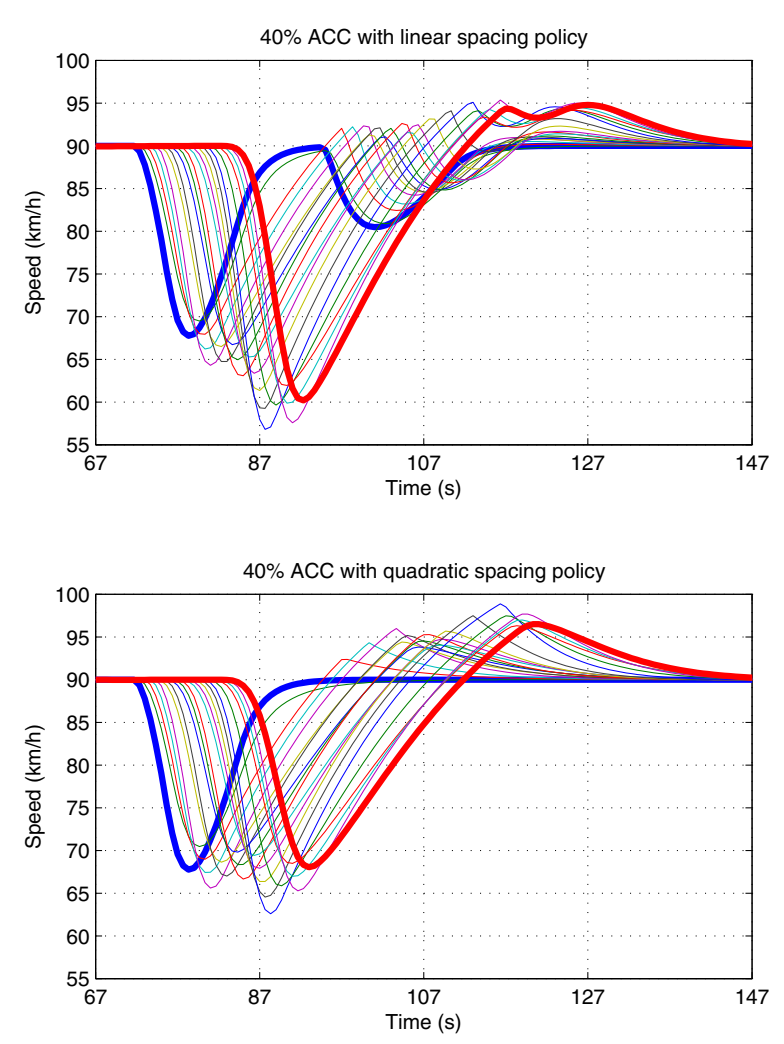

Fig. 4. Speed variation chart of the cars at $\% 40$ ACC penetration in traffic after the shock wave

it is easier to see the influence of the control laws on the cars behind. Both of the spacing policy saved a significant amount of energy as expected because of the achieved smooth traffic flow. The cars with the quadratic spacing policy spent a little less energy compared to the cars with the linear policy since speed is more stable. Even though the difference is little, it can save higher amounts at the continuously disturbed traffic conditions. Furthermore, shorter ranges obtained by quadratic spacing policy may decrease air drag, and reduce the consumption of vehicle [22] [23].

In addition, ACC-equipped vehicles decrease the time travelled significantly. It is also observable in the third figure for the position, HDB travels less than ACC models for the same time interval. Numerically, the last car with ACC decreases time travelled $21.7 \%$ compared to HDB under the same mere penetration conditions as energy consumption.

TABLE III

Percentage of Energy Saved Compared to HDB

\begin{tabular}{ccc}
\hline \hline & Quadratic Policy & Linear Policy \\
\hline \hline All cars in the simulation & $9.3 \%$ & $9.2 \%$ \\
Last ten cars & $11 \%$ & $10.9 \%$ \\
\hline \hline
\end{tabular}


In conclusion, it is found that the quadratic policy has a small gain compared to the linear one in terms of energy consumption and time traveled, but increases significantly string stability and reduces the stop-and-go effect and traffic breakdown. The achieved improvements may become great solutions for today's traffic and environment problems, it can reduce traffic in highways and energy consumption of vehicles significantly. Further analysis of this experiment can be done by increasing the scale of the experiment set-up, i.e. number of vehicles, disturbance etc. or even a step further real life experiment can be done. It is worth to mention that though simulation is done in computer environment, HDB is modeled realistically by using real-life data, and the results of this study highly match with real life. As shockwaves causes higher fuel consumption, travel time and so on, their elimination from traffic prevents these negative effects. Traffic flow stability, another important criteria for traffic stability, can also be investigated for further applications, it requires different experiment set-up than string stability [15].

\section{CONCLUSION}

The ACC-equipped vehicles plays an important role to improve traffic and to reduce energy consumption and travel time. At a penetration rate of $100 \%$ both spacing policies not only prove string stability but also decrease the energy consumption and the time travelled enormously. In comparison with spacing policies, the quadratic one has generated smoother traffic flow compared to the linear, which assures better string stability and lower energy consumption. While having these positive effects, the quadratic one increased the traffic capacity compared to the linear as well. Each spacing policy's impact on string stability also investigated at different penetration rates by combining with HDB. In fact, it is found that the quadratic one is more compatible in mixed traffic situations.

Apart from the results, energy consumption and dynamics of an electric vehicle are explained, formulated and used for the simulation of this study. The formulations and explanations provide a database for prospective electric vehicle researches.

Future studies can cover possible improvements for flexible spacing policies to decrease the energy consumption by optimizing the speed according to changes on road such as curvatures, slopes and traffic lights etc.

Finally, a more advanced version of the ACC system is the cooperative adaptive cruise control (CACC), which also has a vehicle to vehicle communication system [4]. This can further be applied to the proposed ACC model in the paper. In this way, the model performance can be improved since there exists direct information exchange between the vehicles, yet it was not used in this paper to keep the technology as simple as possible to increase the usage of it.

\section{ACKNOWLEDGEMENT}

The authors would like to thank Tim Klemens Schwickart for providing the data, required for the energy calculations and vehicle dynamics, and his supports for this study.

\section{REFERENCES}

[1] F. Viti, S. P. Hoogendoorn, T. P. Alkim, and G. Bootsma, "Driving behavior adaptation to adaptive cruise control: results from a field operational test in the Netherlands," IEEE Intell. Veh. Symp., pp. 745$750,2008$.

[2] J. Pauwelussen and M. Minderhoud, "The effects of deactivation and (re)activation of ACC on driver behaviour analyzed in real traffic," IEEE Intell. Veh. Symp. Proc., pp. 257-262, 2008.

[3] G. Naus, J. Ploeg, R. van de Molengraft and M. Steinbuch, "Explicit MPC design and performance-based tuning of an Adaptive Cruise Control Stop-\&-Go," in Intelligent Vehicles Symposium, IEEE, Eindhoven, 2008.

[4] F. Bu, and C. Chan. "Adaptive and cooperative cruise control." Handbook of Intelligent Vehicles. Springer London, pp. 191-207, 2012.

[5] G. Marsden, M. McDonald and M. Brackstone , "Towards an understanding of adaptive cruise control," in Transportation Research Part C 9, 2001.

[6] A. Vahidi and A. Eskandarian, "Research advances in intelligent collision avoidance and adaptive cruise control," in Intelligent Transportation Systems, IEEE, 2003.

[7] L. Xiao and F. Gao, "A comprehensive review of the development of adaptive cruise control systems," in Vehicle System Dynamics: International Journal of Vehicle Mechanics and Mobility, 2010.

[8] S. Yuki, F. Minoru, K. Macoto, H. Katsuya, N. Akihiro, N. Katsuhiro, T. Shin-ichi and Y. Satoshi, "Traffic jams without bottlenecksexperimental evidence for the physical mechanism of the formation of a jam," New Journal of Physics, 2008.

[9] S. Shladover, D. Su and X. Lu, "Impacts of Cooperative Adaptive Cruise Control on Freeway Traffic Flow," in Journal of the Transportation Research Board, 2012.

[10] I. Ntousakis, I. Nikolos and M. Papageorgiou, "On Microscopic Modelling of Adaptive Cruise Control Systems," Transportation Research Procedia 5, pp. 111-127, 2015.

[11] S. Klinge and R. H. Middleton, "Time Headway Requirements for String Stability of Homogeneous Linear Unidirectionally Connected Systems," Proceedings of the IEEE Conference on Decision and Control, pp. 1992-1997, 2009.

[12] J. Zhou and H. Peng, "Range Policy of Adaptive Cruise Control Vehicles for Improved Flow Stability and String Stability," in IEEE Transactions on Intelligent Transportation Systems, 2005.

[13] P. G. Gipps, "A behavioral car following model for computer simulation," Transport. Res. Board, vol. 15B, pp. 105-111, 1981.

[14] K. Lee, and H. Peng. "Identification and verification of a longitudinal human driving model for collision warning and avoidance systems" International journal of vehicle autonomous systems, pp. 3-17, 2004.

[15] D. Swaroop and K. Rajagopal, "Intelligent cruise control systems and traffic flow stability," Transportation Research Part C, vol. 7, no. 6, p. 329-352, 1999.

[16] B. De Schutter, P. Jeroen , L. Baskar, G. Naus, and H. Nijmeijer. "Hierarchical, intelligent and automatic controls." In Handbook of Intelligent Vehicles, pp. 81-116. Springer London, 2012.

[17] T. Schwickart, H. Voos, J.-R. Hadji-Minaglou, and M. Darouach, "A Fast Model-Predictive Speed Controller for Minimised Charge Consumption of Electric Vehicles," Asian Journal of Control, 18: pp. 133-149, 2016.

[18] T. Schwickart, H. Voos, J.-R. Hadji-Minaglou, M. Darouach, and A. Rosich, "Design and simulation of a real-time implementable energyefficient model-predictive cruise controller for electric vehicles," Journal of the Franklin Institute, vol. 352, no. 2, pp. 603-625, Feb. 2015

[19] A. Kesting, M. Treiber, and D. Helbing, "Enhanced intelligent driver model to access the impact of driving strategies on traffic capacity.," Philos. Trans. A. Math. Phys. Eng. Sci., vol. 368, no. 1928, pp. 45854605,2010

[20] S. Ossen and S. Hoogendoorn, "Car-Following Behavior Analysis from Microscopic Trajectory Data," Transp. Res. Rec., vol. 1934, no. 1, pp. 1321, 2005.

[21] X.-Y. Lu, J. K. Hedrick, and M. Drew, "ACC/CACC-control design, stability and robust performance," Proc. $2002 \mathrm{Am}$. Control Conf., vol. 6, no. Feb. 2002, pp. 4327-4332, 2002.

[22] A. Alam, J. Martensson, and K. H. Johansson, "Experimental evaluation of decentralized cooperative cruise control for heavy-duty vehicle platooning," Control Eng. Pract., vol. 38, pp. 11-25, 2015.

[23] F. Browand, J. McArthur, and C. Radovich, "Fuel Saving Achieved in the Field Test of Two Tandem Trucks," no. June, 2004. 